\title{
MULTI-QUALI: Um modelo de atributos de qualidade para sistemas multi-dispositivo
}

\section{MULTI-QUALI: A quality attributes model for multi-device systems}

Genilda Oliveira de Araujo ${ }^{1}$

Lizandra Garcia Lupi Vergara ${ }^{2}$ 


\section{Resumo}

A disponibilidade de vários dispositivos por pessoa, tais como desktops, tablets e smartphones, abre espaço para o projeto de sistemas que combinam múltiplos dispositivos no suporte à atividade humana. Neste contexto, este artigo propõe um modelo de atributos de qualidade para produtos de software que trata da adequação da distribuição de tarefas entre dispositivos e da qualidade das interfaces em cada um deles. Sua construção foi feita a partir de uma revisão sistemática de literatura sobre ergonomia, usabilidade e experiência do usuário neste tipo de sistema. Sua principal contribuição é sistematizar parâmetros que servem de apoio à concepção e à avaliação de sistemas multi-dispositivo.

Palavras-chave: design de interação, usabilidade, ergonomia, multi-dispositivo.

\section{Abstract}

The availability of multiple devices per person, such as desktops, tablets and smartphones, makes room for the design of systems that combine multiple devices to support human activity. In this context, this paper proposes a model of quality attributes for software products that deals with the appropriateness of task distribution between devices and the quality of interfaces on each device. Its construction was based on a systematic literature review on ergonomics, usability and user experience in this type of system. Its main contribution is to systematize parameters that support the design and evaluation of multi-device systems.

Key-words: interaction device, usability, ergonomics, multi-device.

ISSN: 2316-7963

${ }^{1}$ Doutoranda em Engenharia, UFSC (genilda@gmail.com)

2 Doutora em Engenharia, UFSC (I.vergara@ufsc.br) 


\section{Introdução}

Os últimos anos foram marcados pela popularização do uso de dispositivos móveis como smartphones e tablets. Além deles, wearables como relógios, pulseiras e óculos começaram a integrar a vida cotidiana das pessoas. Esta ampliação no número e na variedade de dispositivos traz novos desafios para a concepção de produtos de software.

$\mathrm{Na}$ era do computador pessoal, as pessoas geralmente tinham acesso a um dispositivo, o computador, e o utilizavam como estação de trabalho para realizar suas tarefas profissionais e pessoais (HARPER, 2008). Neste período, os sistemas eram concebidos considerando o uso deste único dispositivo e a avaliação de qualidade era realizada dentro deste escopo. Atualmente, a disponibilidade de múltiplos dispositivos por pessoa permite que as tarefas sejam realizadas por meio de diferentes dispositivos. Desta forma, uma pessoa pode iniciar uma tarefa em um dispositivo e finalizá-la em outro, ou ainda usá-los simultaneamente. Por exemplo, é possível iniciar a redação de um texto no desktop e finalizá-la no smartphone, ou, ainda, usar um smartphone como controle remoto para uma apresentação de slides exibida no desktop.

Segundo Zagermann et al. (2017), este novo cenário amplia o escopo da Interação Homem-Computador (IHC), que deixa de abordar apenas o uso isolado de dispositivos e passa também a tratar do uso de conjuntos de dispositivos. Assim, abre-se um campo de pesquisa focado em configurações de múltiplos dispositivos, que dão suporte às tarefas com base em combinações, relações e transferências entre dispositivos.

Considerando estas questões, este artigo propõe um modelo de atributos de qualidade para produtos de software que trata da adequação da distribuição de tarefas entre dispositivos e da qualidade das interfaces em cada um deles. Sua construção foi feita a partir de uma revisão sistemática de literatura sobre ergonomia, usabilidade e experiência do usuário neste tipo de sistema. Sua principal contribuição é sistematizar parâmetros que servem de apoio à concepção e à avaliação de sistemas multidispositivo.

\section{Fundamentação Teórica}

Esta seção do artigo descreve a interação multi-dispositivo e suas variações, servindo de base para a compreensão dos atributos propostos. Por ser uma área recente da IHC, sua terminologia não está difundida. Desta forma, é necessário realizar um alinhamento de termos.

Como ponto de partida, são descritos os pares de termos dispositivo/plataforma e multi/cross. Em ambos os pares, há uma diferenciação no que diz respeito à abrangência. No primeiro par, o termo dispositivo é mais genérico e designa um aparelho formado por um hardware (equipamento físico) e um sistema operacional (software como Windows, Mac OS X, iOS ou Android). Assim, qualquer notebook, tablet ou smartphone é um dispositivo. Por outro lado, plataforma designa uma combinação específica de hardware e sistema operacional (FLORINS; VANDERDONCKT, 2004). Por exemplo, o Apple iPhone XS e o Samsung Galaxy S9 são dispositivos de plataformas diferentes. No segundo par, o termo multi é mais genérico e descreve a existência de vários. Por sua vez, o termo cross descreve um subcaso de multi no qual os vários dispositivos são usados de forma relacionada para realizar uma mesma tarefa.

Como resultado das combinações dos termos, tem-se quatro denominações

HFD, v.9, n 17, p. 75-95, junho 2020 
possíveis: multi-device, multi-platform, cross-device e cross-platform. A primeira é a mais abrangente e engloba as demais. Assim, a denominação multi-device, ou multidispositivo, é usada neste artigo para representar qualquer sistema que envolve vários dispositivos. Os subtipos são descritos a seguir e sintetizados na figura 1. Como não foi encontrada tradução de cross-device ou cross-platform para português, optou-se por manter a padronização de idioma e as denominações são sempre usadas em inglês.

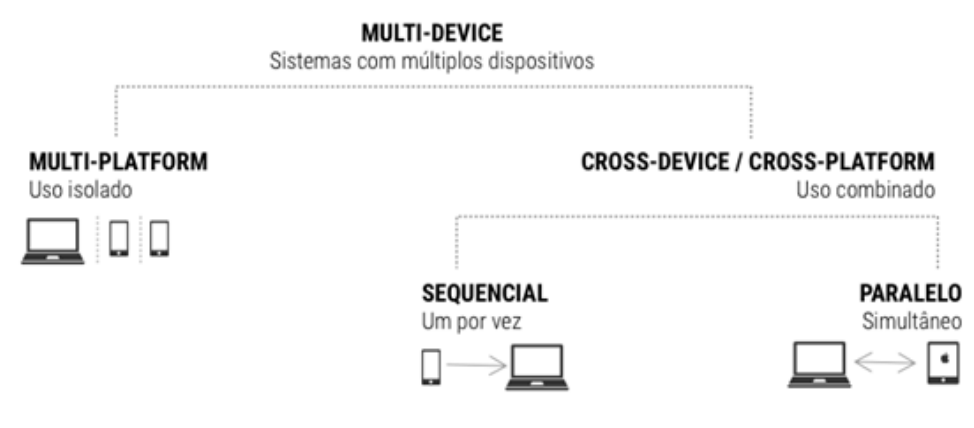

Figura 1 - Variações multi-device (fonte: autores).

O termo multi-platform designa sistemas compatíveis com mais de uma combinação de hardware e sistema operacional. Assim, aplicativos como o Adobe Photoshop são multi-platform, pois estão disponíveis em computadores com sistema operacional Windows e Mac Os X. Neste caso, a multiplicidade tem a função de aumentar os canais de acesso ao sistema, sendo, por isso, chamado de multichanneled (WÄLJAS et al., 2010) ou multi-target (CALVARY et al., 2003). Neste caso, o foco não é o uso conjunto de dispositivos, mas o uso isolado em diferentes plataformas. Segundo Levin (2014), o projeto deste tipo de sistema foca em oferecer a mesma experiência básica em todos os dispositivos, mantendo conteúdo, fluxo, estrutura e funções básicas.

Para designar sistemas que envolvem vários dispositivos para realizar uma tarefa (WÄLJAS et al., 2010), usa-se os termos cross-device ou cross-platform. A diferença entre eles está na amplitude. O cross-device abarca qualquer sistema com mais de um dispositivo, mesmo que sejam da mesma plataforma. Assim, um sistema que combina dois iPads é cross-device, mas não é cross-platform. Este termo se refere a sistemas que envolvem pelo menos dois dispositivos de plataformas distintas (WÄLJAS et al., 2010). Assim, qualquer sistema cross-platform é cross-device, mas o inverso não é verdadeiro. Em função disso, este trabalho adota a denominação mais genérica, que é o termo cross-device.

Tanto o cross-device quanto o cross-platform podem ser sequencial ou simultâneo. Segundo Jokela et al. (2015), no sequencial há a troca de dispositivo durante a tarefa. Como exemplo, pode-se citar WhatsApp e Telegram, que mantém a sincronia e permitem que os usuários continuem as conversas de onde pararam ao trocar de dispositivo. De acordo com Levin (2014), o projeto sequencial foca na continuidade da ação e o sistema deve oferecer recursos para facilitar a migração de um dispositivo para o próximo. No simultâneo, ocorre o uso paralelo relacionado (JOKELA; OJALA; OLSSON, 2015), no qual o usuário envolve dois ou mais dispositivos em uma tarefa, distribuindo-a entre eles. Por exemplo, no Google Apresentações, é possível utilizar o smartphone como controle remoto para uma apresentação exibida no desktop. Segundo Levin 
(2014), o projeto simultâneo visa oferecer uma experiência integrativa de dispositivos que colaboram como um grupo conectado e controlam um ao outro.

Além de terminologias para os tipos de sistemas, há terminologias para os tipos de interface multi-device. O termo multiple user interfaces descreve um sistema que, segundo Nguyen et al. (2016), visa oferecer uma experiência de usuário similar em vários dispositivos. Pelo foco na consistência, estão geralmente ligadas ao multi-platform. Outro termo importante é migratory user interface, que designa um aplicativo que pode transferir a atividade do usuário entre dispositivos, permitindo que inicie a atividade em um dispositivo e termine em outro (DEES, 2011). Esta característica é essencial para sistemas cross-device sequenciais. Por fim, as distributed user interfaces possuem partes da interface distribuídas por pelo menos dois dispositivos (PATERNÒ; SANTORO, 2012), sendo comumente associados ao cross-device paralelo.

\section{Metodologia}

Com o propósito de identificar atributos de qualidade para sistemas multi-device, foi realizada uma revisão bibliográfica de modo sistemático que seguiu a metodologia de Kitchenham e Charters (2007). Esta abordagem sugere cinco passos: (a) definição da pergunta de pesquisa; (b) definição da estratégia de busca; (c) busca; (d) seleção de resultados primários; (e) extração de dados.

A pergunta de pesquisa definida foi: "quais são os atributos de qualidade desejados para sistemas que envolvem o uso de múltiplos dispositivos?". Como estratégia de busca, foram selecionadas as seguintes palavras-chave: "usability" or "user experience" or "ergonomics" or "human factors") AND ("multi-device" or "cross-device" or "crossplatform" or "multi-platform" or "multiple user interfaces" or "migratory user interfaces" or "distributed user interfaces"). O primeiro grupo de palavras chave foi selecionado para abordar a questão dos atributos de qualidade. O segundo grupo foca no multidevice e faz uso de todas as terminologias descritas na fundamentação teórica.

A busca foi realizada nas bases de dados SCOPUS, ACM Digital Library e Web of Science. Foram incluídos na pesquisa artigos de revistas e de conferências, bem como capítulos de livro. Optou-se por não limitar o período de publicação. Na seleção dos resultados primários, foram incluídos estudos que:

- 11: Descrevem aspectos que influenciam na qualidade multi-device;

Foram excluídos estudos nos quais:

-El: As palavras-chave foram apenas brevemente citadas;

-E2: Há foco nas questões tecnológicas para desenvolvimento do sistema.

A tabela 1 apresenta um panorama quantitativo da revisão, que mostra uma grande quantidade de artigos descartados. A razão para isso é que esta é uma área recente na qual ainda predominam estudos focados nas questões técnicas para a construção destes tipos de sistemas.

Tabela 1. Panorama quantitativo da revisão sistemática (fonte:autores).

\begin{tabular}{lccc}
\hline Data Base & Resultados & Não Repetidos & Selecionados \\
\hline SCOPUS & 378 & 374 & 18 \\
ACM Digital & 63 & 28 & 1 \\
Web of Science & 91 & 60 & 2
\end{tabular}

HFD, v.9, n 17, p. 75-95, junho 2020 


\section{Resultados}

Os resultados da extração e análise de dados estão organizados em dois tópicos: (4.1) atributos de qualidade multi-device; (4.2) modelo descritivo para qualidade multidevice.

\subsection{Atributos de qualidade multi-device}

Foram identificados 21 trabalhos que descrevem aspectos que influenciam na qualidade multi-device, conforme mostrado na tabela 2.

Tabela 2. Trabalhos que abordam a qualidade multi-device (fonte:autores).

\begin{tabular}{|c|c|}
\hline AUTORES & FATORES, REQUISITOS OU HEURÍSTICAS \\
\hline (DENIS; KARSENTY, 2003) & Consistência, transparência, adaptabilidade \\
\hline (SEFFAH; FORBRIG; JAVAHERY, 2004) & $\begin{array}{l}\text { Usabilidade vertical e horizontal, adaptabilidade da estrutura da } \\
\text { tarefa, escalabilidade da informação, significação visual, estilo de } \\
\text { interação, restrições e capacidades da plataforma, comportamento, } \\
\text { disponibilidade }\end{array}$ \\
\hline (LUYTEN; CONINX, 2005) & Completude, continuidade \\
\hline (ÖQUIST; GOLDSTEIN; CHINCHOLLE, 2005) & Portabilidade, atenção, gerenciabilidade, apreensibilidade. \\
\hline (RINGBAUER, 2005) & Consistência \\
\hline (ALSOS; SVANÆES, 2006) & $\begin{array}{l}\text { Mudança de foco, ergonomia do arranjo físico, ocultação de infor- } \\
\text { mações }\end{array}$ \\
\hline (HUTCHINGS; PIERCE, 2006) & Privacidade, qualidade da entrada e saída \\
\hline (SEGERSTÅHL; OINAS-KUKKONEN, 2007) & $\begin{array}{l}\text { Continuidade, habilidades técnicas, personalização, coerência se- } \\
\text { mântica }\end{array}$ \\
\hline (SEGERSTÅHL, 2008) & Heterogeneidade, interoperabilidade, consistência \\
\hline (TUNGARE; PÉREZ-QUINONES, 2009) & Transições \\
\hline (WÄLJAS et al., 2010) & Composição, continuidade, consistência \\
\hline (DEES, 2011) & Consistência \\
\hline (SKOV et al., 2015) & $\begin{array}{l}\text { Consistência, divisão entre dispositivos pessoais e compartilhados, } \\
\text { sincronização }\end{array}$ \\
\hline $\begin{array}{l}\text { (MAJRASHI; HAMILTON; L. UITDENBOGERD, } \\
\text { 2016) }\end{array}$ & $\begin{array}{l}\text { Consistência, fluência, organização, leiturabilidade, reconhecimen- } \\
\text { to, transparência }\end{array}$ \\
\hline (CELENTANO; DUBOIS, 2017) & $\begin{array}{l}\text { Arranjo físico, manipulação do dispositivo, envio de informações, } \\
\text { interação no pequeno e interação no grande }\end{array}$ \\
\hline $\begin{array}{l}\text { (MAJRASHI; HAMILTON; UITDENBOGERD, } \\
\text { 2017) }\end{array}$ & Consistência, fluência, organização, leiturabilidade, reconhecimento \\
\hline (NEATE; EVANS; JONES, 2017) & Atenção \\
\hline (SHIN; BIOCCA, 2017) & Acessibilidade, disponibilidade, confiabilidade, portabilidade \\
\hline (ZAGERMANN et al., 2017) & Distribuição espacial \\
\hline $\begin{array}{l}\text { (MAJRASHI; HAMILTON; UITDENBOGERD, } \\
\text { 2018) }\end{array}$ & Consistência, controlabilidade, conectividade \\
\hline (AABEL; ABEYWARNA, 2018) & $\begin{array}{l}\text { Comportamento consistente, branding, design de informação e } \\
\text { conteúdo, canais apropriados, audiência apropriada, uso das forças } \\
\text { dos canais, experiência fluída }\end{array}$ \\
\hline
\end{tabular}


A análise dos trabalhos selecionados permitiu identificar que não há padronização na nomenclatura adotada. Alguns trabalhos abordam fatores de qualidade, outros métricas, outros requisitos e outros ainda heurísticas. Em função disso, optou-se por consolidar os diferentes aspectos descritos na forma de atributos de qualidade, tomando como base a nomenclatura proposta pela norma ISO/IEC 25010 (ISO, 2011).

Esta é a norma mais recente a tratar do modelo de qualidade para produtos de software, substituindo a norma ISO/IEC 9126-1. Descreve dois tipos de atributos: de qualidade do produto e de qualidade no uso. Este artigo faz uso da nomenclatura para atributos de qualidade do produto, que influenciam nos atributos de qualidade no uso.

As descrições dos aspectos abordados pelos autores foram analisadas e estes aspectos foram agrupados e associados a um atributo de qualidade, conforme mostrado na tabela 3. Foram identificados cinco atributos e nove sub-atributos de qualidade do produto impactados pela interação multi-device. Cada um deles é discutido a seguir.

Tabela 3. Atributos de qualidade multi-device e aspectos relacionados (fonte:autores).

\begin{tabular}{|c|c|}
\hline ATRIBUTOS & FATORES, REQUISITOS OU HEURÍSTICAS RELACIONADOS \\
\hline $\begin{array}{l}\text { ADEQUAÇÃO FUN- } \\
\text { CIONAL }\end{array}$ & $\begin{array}{l}\text { Composição (WÄLJAS et al., 2010) } \\
\text { Configuração (MAJRASHI; HAMILTON; UITDENBOGERD, 2017) } \\
\text { Interoperabilidade (SEGERSTÅHL, 2008) } \\
\text { Organização (MAJRASHI; HAMILTON; L. UITDENBOGERD, 2016) }\end{array}$ \\
\hline $\begin{array}{l}\text { Adequação aos dis- } \\
\text { positivos }\end{array}$ & $\begin{array}{l}\text { Canais apropriados (AABEL; ABEYWARNA, 2018) } \\
\text { Disponibilidade do serviço (SEFFAH; FORBRIG; JAVAHERY, 2004) }\end{array}$ \\
\hline $\begin{array}{l}\text { Adequação aos usuá- } \\
\text { rios }\end{array}$ & $\begin{array}{l}\text { Adaptação ao perfil do usuário (DENIS; KARSENTY, 2003) } \\
\text { Adequação à audiência (AABEL; ABEYWARNA, 2018) } \\
\text { Habilidades técnicas e personalização (SEGERSTÅHL; OINAS-KUKKONEN, 2007) }\end{array}$ \\
\hline $\begin{array}{l}\text { Completude funcio- } \\
\text { nal }\end{array}$ & Completude (LUYTEN; CONINX, 2005) \\
\hline PORTABILIDADE & Portabilidade (SHIN; BIOCCA, 2017) \\
\hline Adaptabilidade & $\begin{array}{l}\text { Adaptabilidade da estrutura da tarefa, escalabilidade da informação, significação visual, } \\
\text { estilo de interação, restrições e capacidades da plataforma (SEFFAH; FORBRIG; JAVAHERY, } \\
\text { 2004). } \\
\text { Uso das forças do canal (AABEL; ABEYWARNA, 2018) }\end{array}$ \\
\hline USABILIDADE & $\begin{array}{l}\text { Interação no pequeno e interação no grande (CELENTANO; DUBOIS, 2017). } \\
\text { Usabilidade vertical e horizontal (SEFFAH; FORBRIG; JAVAHERY, 2004). }\end{array}$ \\
\hline Operacionalidade & $\begin{array}{l}\text { Atenção (NEATE; EVANS; JONES, 2017) } \\
\text { Controlabilidade (MAJRASHI; HAMILTON; UITDENBOGERD, 2018) } \\
\text { Mudança de foco, ergonomia do arranjo físico (ALSOS; SVAN/ES, 2006) } \\
\text { Gerenciabilidade, atenção (ÖQUIST; GOLDSTEIN; CHINCHOLLE, 2005) } \\
\text { Arranjo físico (CELENTANO; DUBOIS, 2017) } \\
\text { Qualidade da entrada e saída (HUTCHINGS; PIERCE, 2006) } \\
\text { Distribuição espacial (ZAGERMANN et al., 2017) }\end{array}$ \\
\hline Apreensibilidade & $\begin{array}{l}\text { Heterogeneidade (SEGERSTÅHL, 2008) } \\
\text { Apreensibilidade (MAJRASHI; HAMILTON; L. UITDENBOGERD, 2016; MAJRASHI; HAMILTON; } \\
\text { UITDENBOGERD, 2017; ÖQUIST; GOLDSTEIN; CHINCHOLLE, 2O05) } \\
\text { Transparência (DENIS; KARSENTY, 2003; MAJRASHI; HAMILTON; L. UITDENBOGERD, 2016) }\end{array}$ \\
\hline
\end{tabular}




\begin{tabular}{|c|c|}
\hline $\begin{array}{l}\text { Consistência entre } \\
\text { dispositivos }\end{array}$ & $\begin{array}{l}\text { Comportamento (SEFFAH; FORBRIG; JAVAHERY, 2004). } \\
\text { Consistência (DEES, 2011; DENIS; KARSENTY, 2003; MAJRASHI; HAMILTON; L. UITDENBO- } \\
\text { GERD, 2016; MAJRASHI; HAMILTON; UITDENBOGERD, 2017, 2018; RINGBAUER, 2005; SE- } \\
\text { GERSTÅHL, 2008; SKOV et al., 2015; WÄLJAS et al., 2010) } \\
\text { Comportamento consistente, branding, design de informação e conteúdo (AABEL; } \\
\text { ABEYWARNA, 2018) } \\
\text { Envio de informação (CELENTANO; DUBOIS, 2017) } \\
\text { Coerência semântica (SEGERSTÅHL; OINAS-KUKKONEN, 2007) }\end{array}$ \\
\hline $\begin{array}{l}\text { Interoperabilidade } \\
\text { entre dispositivos }\end{array}$ & $\begin{array}{l}\text { Continuidade (DENIS; KARSENTY, 2003; LUYTEN; CONINX, 2005; SEGERSTÅHL; OINAS- } \\
\text {-KUKKONEN, 2007; WÄLJAS et al., 2010) } \\
\text { Fluência (MAJRASHI; HAMILTON; L. UITDENBOGERD, 2016; MAJRASHI; HAMILTON; UIT- } \\
\text { DENBOGERD, 2017) } \\
\text { Reconhecimento (MAJRASHI; HAMILTON; L. UITDENBOGERD, 2016; MAJRASHI; HAMILTON; } \\
\text { UITDENBOGERD, 2017) } \\
\text { Experiência fluída (AABEL; ABEYWARNA, 2018) } \\
\text { Sincronização (SKOV et al., 2015) } \\
\text { Transições (TUNGARE; PÉREZ-QUINONES, 2009) }\end{array}$ \\
\hline CONFIABILIDADE & Confiabilidade (SHIN; BIOCCA, 2017) \\
\hline Disponibilidade & $\begin{array}{l}\text { Acessibilidade, disponibilidade (SHIN; BIOCCA, 2017) } \\
\text { Conectividade (MAJRASHI; HAMILTON; UITDENBOGERD, 2018) }\end{array}$ \\
\hline SEGURANÇA & $\begin{array}{l}\text { Privacidade (HUTCHINGS; PIERCE, 2006) } \\
\text { Ocultação de informações (ALSOS; SVAN/ES, 2006) } \\
\text { Divisão entre dispositivos pessoais e compartilhados (SKOV et al., 2015) }\end{array}$ \\
\hline
\end{tabular}

\subsubsection{Adequação Funcional}

Este atributo indica a capacidade do produto de prover funções que atendam às necessidades dos usuários sob condições de uso especificadas (ISO, 2011). No multidevice, é mais complexo obter essa adequação de funções. Os diferentes dispositivos possuem características interativas que os tornam mais ou menos compatíveis com a realização de cada tarefa (PATERNÒ, 2009). Para o autor, por exemplo, há uma distinção entre o que é significativo fazer com um desktop e com um smartphone. Em função disso, a definição das funções envolve duas etapas (SEFFAH; FORBRIG; JAVAHERY, 2004). A primeira é a criação de um modelo geral das tarefas que serão suportadas pelo produto. A seguir, deve-se distribuir essas tarefas entre os dispositivos, dando origem a um modelo por dispositivo (SEFFAH; FORBRIG; JAVAHERY,2004), conforme mostrado na figura 2. Neste modelo, algumas tarefas podem ser suportadas isoladamente por todos os dispositivos (ex: tarefa 1), algumas tarefas podem ser executadas exclusivamente em um dispositivo (ex: tarefas 2 e 3 ) e outras tarefas podem envolver o uso combinado de dispositivos (ex: tarefa 4). Mesmo em tarefas suportadas isoladamente (ex: tarefa 1), pode haver sincronização de dados entre dispositivos, permitindo a troca de dispositivo.

A arquitetura funcional (SEGERSTÅHL, 2008) ou composição do sistema (WÄLJAS et al., 2010) distingue o papel dos dispositivos no sistema e define como as funções e os conteúdos serão distribuídos entre eles. Por exemplo, em um aplicativo de e-mail, smartwatches podem focar em emitir notificações, smartphones podem ser usados para leitura e respostas rápidas, ao passo que desktops podem permitir a gestão mais completa da caixa de entrada e a redação de mensagens longas. Assim, a adequação funcional multi-device está ligada à organização apropriada das funções e conteúdos 
entre os dispositivos (MAJRASHI; HAMILTON; L. UITDENBOGERD, 2016; MAJRASHI; HAMILTON; UITDENBOGERD, 2017). Este processo também inclui, quando pertinente, o planejamento das tarefas que envolvem o uso de mais de um dispositivo (MAJRASHI; HAMILTON; L. UITDENBOGERD, 2016), estabelecendo funcionalidades cross-device.

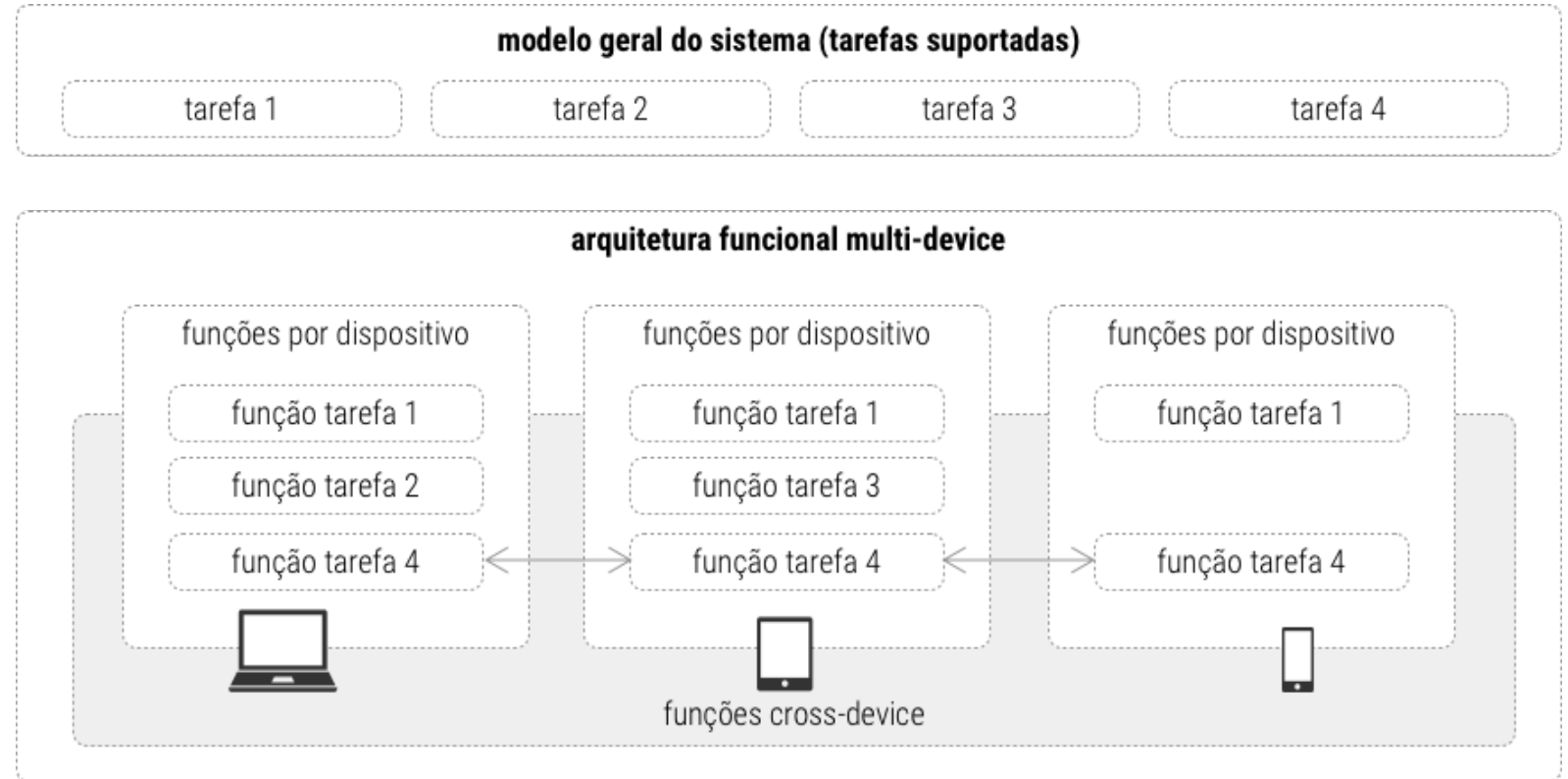

Figura 2 - Arquitetura funcional multi-device (fonte: autores).

Considerando a complexidade deste processo, este artigo propõe dois subatributos para auxiliar na concepção da arquitetura funcional multi-device: adequação aos dispositivos e adequação aos usuários. Além disso, também sugere uma expansão na descrição do atributo completude funcional. Estes três sub-atributos são descritos a seguir.

A adequação aos dispositivos se relaciona à compreensão de que nem todas as funções e conteúdos precisam estar disponíveis em todos os dispositivos (SEFFAH; FORBRIG; JAVAHERY, 2004). Por exemplo, digitação no smartwatch não é viável pelo tamanho da tela. De acordo com Aabel e Abeywarna (2018), as funções devem ser disponibilizadas em dispositivos apropriados à tarefa que adicionem valor à sua execução. Para os autores, valor é a diferença entre benefícios e custos da experiência.

A adequação aos usuários aborda o fato de que certos dispositivos podem ser adequado à tarefa, mas não serem compatíveis com o perfil dos usuários. Por exemplo, podem requerer habilidades técnicas especializadas para que o usuário se beneficie integralmente do sistema (SEGERSTÅHL; OINAS-KUKKONEN, 2007). Assim, é importante escolher dispositivos relevantes e adequados aos usuários, considerando contexto de uso, conhecimento técnico, habilidades, limitações e preferências (AABEL; ABEYWARNA, 2018). Além disso, deve-se preferencialmente adotar dispositivos familiares (DENIS; KARSENTY, 2003) e usar estratégias de personalização (SEGERSTÅHL; OINAS-KUKKONEN, 2007)

A completude funcional foca na capacidade das funções abrangerem todas as tarefas e todos os objetivos do usuário (ISO, 2011). No multi-device, esta completude pode ser comprometida pela estratégia de distribuição das funções entre dispositivos. Segundo Luyten e Coninx (2005), é preciso oferecer ao usuário, no momento 
requerido, funções para execução de todas as tarefas desejadas, independentemente do dispositivo. Assim, mesmo que não haja funções para uma certa tarefa em todos os dispositivos, no momento da execução, o usuário deve dispor de pelo menos um dispositivo compatível. Por exemplo, em um sistema de e-mail, caso o desktop não esteja disponível, o usuário pode usar o smartphone para escrever uma mensagem, mesmo que não seja o melhor dispositivo para a tarefa.

\subsubsection{Portabilidade}

Define grau de eficácia e eficiência com o qual um produto pode ser transferido de um hardware ou ambiente operacional para outro (ISO, 2011). Ou seja, diz respeito à possibilidade de usar o produto em diferentes plataformas para os mesmos fins funcionais (SHIN; BIOCCA, 2017). O ideal seria portar o produto para a maior quantidade possível de plataformas, permitindo seu uso generalizado. Um sub atributo essencial para portabilidade é a adaptabilidade.

Conformea norma ISO/IEC25010 (ISO,2011), adaptabilidade consistena capacidade do produto ser adaptado para hardware, software ou ambientes operacionais diferentes. Em sistemas multi-device, esse é um dos pontos centrais a serem tratados. Segundo Seffah et al. (2004), devido a variações nas restrições e capacidades dos dispositivos, a interface do usuário pode ser diferente entre eles. Para definir o tipo de adaptação a ser feito, devem ser explorados os pontos fortes de cada dispositivo, de modo a incorporar seus recursos nativos e adotar as melhores práticas (AABEL; ABEYWARNA, 2018).

A adaptação pode se basear em ajustes da estrutura da tarefa entre os dispositivos (SEFFAH; FORBRIG; JAVAHERY, 2004). Uma possibilidade é oferecer, no dispositivo com mais restrições, acesso parcial a uma função complexa, eliminando passos e informações que não podem ser apresentados. Outra possibilidade é decompor a tarefa de forma diferente. Por exemplo, em um desktop, com mais espaço de visualização, todas os passos podem ser apresentadas de uma vez. Em um smartphone, podem ser apresentados em duas ou mais telas em função da limitação de espaço, conforme mostra a figura 3.

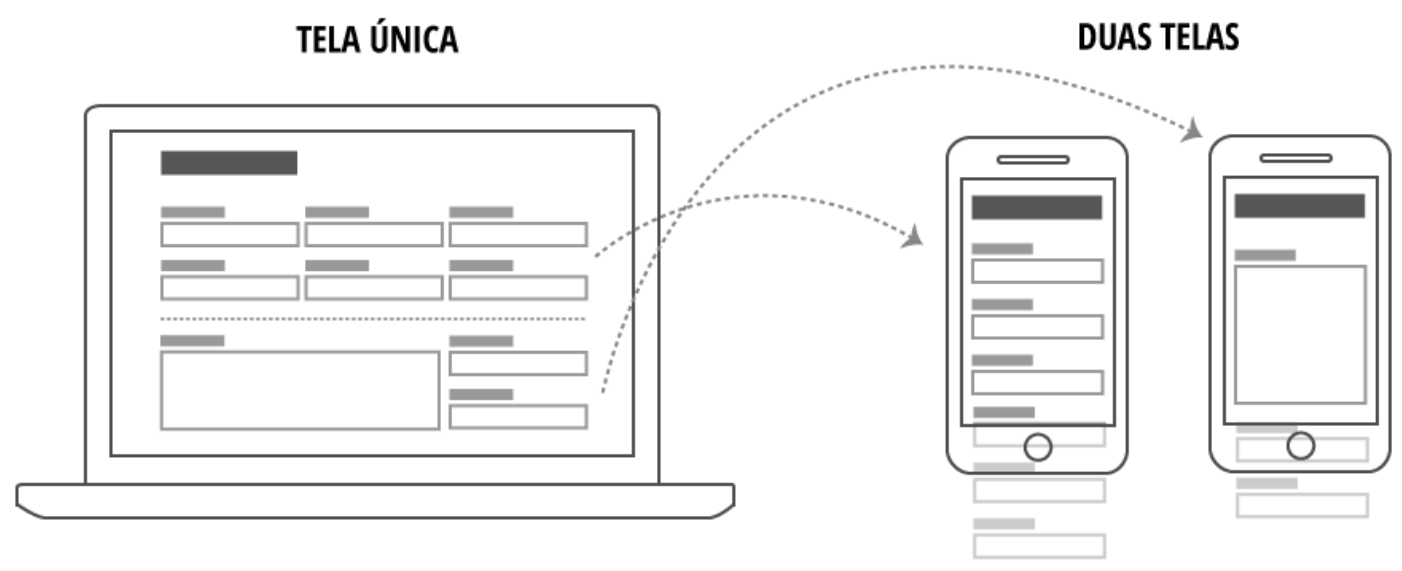

Figura 3 - Adaptação na estrutura da tarefa (fonte: autores).

Além do ajuste da estrutura da tarefa, é possível modificar a forma de apresentação e o estilo de interação com cada passo da tarefa (SEFFAH; FORBRIG; JAVAHERY, 2004). 
Por exemplo, pode-se usar componentes diferentes entre aplicativos dos sistemas Android e iOS ou entre dispositivos que têm variação no tamanho de tela. Por exemplo, um componente de abas, que permite navegar entre seções de uma tela em um desktop, requer grande espaço horizontal, podendo não se adequar a smartphones. Neste caso, pode ser substituído por algum outro componente de seleção como um combobox, conforme ilustrado na figura 4.

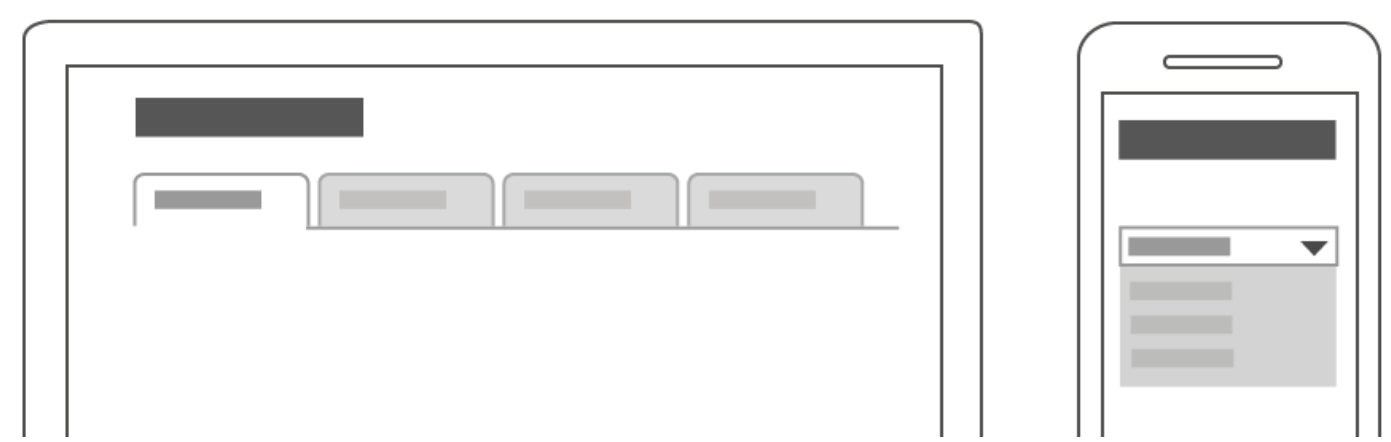

Figura 4 - Adaptação nos componentes de interação na interface (fonte: autores).

\subsubsection{Usabilidade}

Descreve o grau com o qual um produto pode ser usado por usuários específicos para atingir metas especificadas com eficácia, eficiência e satisfação em um contexto específico de uso (ISO, 2011). No multi-device, pode ser dividida em duas dimensões: usabilidade vertical e usabilidade horizontal (SEFFAH; FORBRIG; JAVAHERY, 2004), conforme mostrado na figura 5. A primeira se refere aos requisitos de usabilidade específicos para cada dispositivo. Está relacionada à concepção da interação com cada dispositivo, definindo as sequências de operações que o usuário executa em cada um deles a fim de atingir um certo objetivo (CELENTANO; DUBOIS, 2017). Por outro lado, a usabilidade horizontal está relacionada à qualidade de tarefas que envolvem o uso de vários dispositivos, seja de modo sequencial ou simultâneo. Este caso envolve a concepção de uma visão mais global que relaciona tarefas e dispositivos em um procedimento (CELENTANO; DUBOIS, 2017).

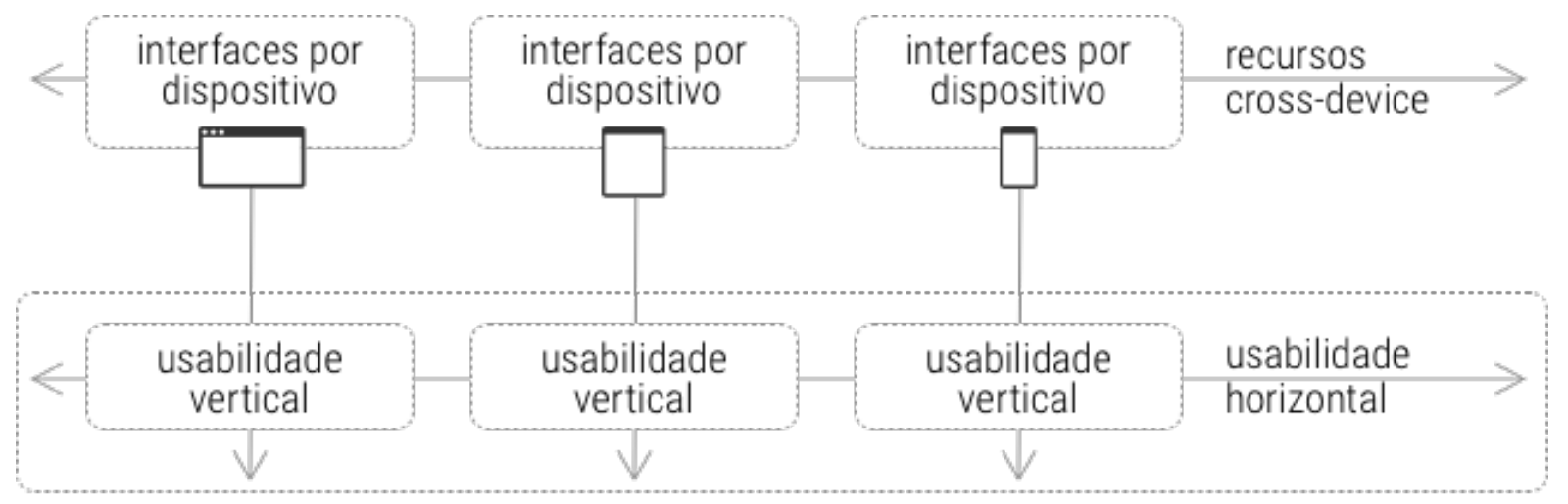

experiência do usuário

Figura 5 - Usabilidade vertical e horizontal (fonte: autores). 
Para tratar essas questões, propõe-se ampliar a descrição de dois sub-atributos de usabilidade: operacionalidade e apreensibilidade. Estes dois atributos relacionamse tanto à usabilidade vertical quanto horizontal. Além disso, foram propostos dois sub-atributos focados na usabilidade horizontal: consistência entre dispositivos e interoperabilidade entre dispositivos.

A operacionalidade descreve o grau em que um produto possui características que facilitam sua operação e seu controle (ISO, 2011). No multi-device, define a capacidade do sistema de permitir que o usuário manipule as diferentes interfaces (ÖQUIST; GOLDSTEIN; CHINCHOLLE, 2005) sem erros interativos (MAJRASHI; HAMILTON; UITDENBOGERD, 2018). Depende de três aspectos: (a) modalidades interativas; (b) nível de atenção que o usuário pode dispensar; (c) ergonomia do arranjo físico. Os três são descritos a seguir.

O termo modalidade interativa se refere ao tipo de canal de comunicação usado por um dispositivo computacional para enviar ou receber informação (NIGAY; COUTAZ, 1993). Dispositivos como desktops tradicionalmente usam interfaces visuais controladas por elementos apontadores como mouse. Dispositivos mais recentes incorporam, por exemplo, o uso de gestos em telas sensíveis ao toque, comandos de voz, bem como detecção de presença, sinais vitais e resposta a comandos de movimento (VAN DAM, 2000). No âmbito da operacionalidade, os tipos de modalidades influenciam na performance e na precisão com os quais os usuários manipulam as interfaces. Segundo Hutchings e Pierce (2006), os usuários muitas vezes selecionam o dispositivo considerando a quantidade de leitura, rolagem, exibição de mídia e entrada de texto envolvida na tarefa. Além disso, o nível de controle de cada modalidade pode variar de acordo com a estabilidade provida pelo dispositivo e sua situação de uso (ÖQUIST; GOLDSTEIN; CHINCHOLLE, 2005): (a) estável com duas mãos (ex: sentado usando o desktop); (b) instável com duas mãos (ex: tablet em pé); (c) instável com uma mão (ex: smartphone em pé).

No que diz respeito ao nível de atenção, pode-se destacar três variações: primária, secundária ou mínima (ÖQUIST; GOLDSTEIN; CHINCHOLLE, 2005). Tais variações têm relação com a natureza da tarefa e com a situação de uso. Por exemplo, ao consultar um trajeto em um mapa, o usuário pode dispensar diferentes níveis de atenção quando realiza esta tarefa em casa e enquanto dirige. Como consequência, deve-se identificar a situação de uso mais frequente e adaptar a complexidade da interface a esta situação. Ainda no âmbito da atenção, é preciso observar a relação custo/benefício do uso simultâneo de dispositivos. Para se envolver com mais de um dispositivo, os usuários precisam gerenciar sua atenção, ajustando frequentemente seu foco (ALSOS; SVAN/ES, 2006), fato que eleva o esforço cognitivo e pode reduzir a operacionalidade (NEATE; EVANS; JONES, 2017). Por fim, no caso de uso simultâneo, a operacionalidade também é impactada pelo arranjo físico dos dispositivos (ALSOS; SVAN/ES, 2006). Assim, um planejamento inadequado da distribuição espacial pode causar interrupção da tarefa (ZAGERMANN et al., 2017) e desconforto pela necessidade de trocar de postura ou posicionamento durante a interação (CELENTANO; DUBOIS, 2017).

Outro sub atributo da usabilidade é a apreensibilidade, que diz respeito à capacidade do produto de software de possibilitar ao usuário aprender sua aplicação (ISO, 2011). A heterogeneidade dos dispositivos pode ampliar bastante os requisitos de aprendizagem para os usuários, o que inclui, por exemplo, fundamentos para o uso do PC, da web e de dispositivos móveis (SEGERSTÅHL, 2008). Assim, em sistemas multi-device, a apreensibilidade deve considerar o grau com o qual as interfaces de 
cada dispositivo precisam ser aprendidas separadamentes (MAJRASHI; HAMILTON; L. UITDENBOGERD, 2016; MAJRASHI; HAMILTON; UITDENBOGERD, 2017; ÖQUIST; GOLDSTEIN; CHINCHOLLE, 2005). Outro aspecto que pode ser associado a este atributo é a construção de uma representação mental do sistema por parte do usuário. No multidevice, essa questão é mais complexa, pois envolve a compreensão do papel de cada dispositivo no processo de ação. O sistema deve ser transparente (DENIS; KARSENTY, 2003), de modo que a interface permita ao usuário entender quais dados e funções estão disponíveis em cada dispositivo (MAJRASHI; HAMILTON; L. UITDENBOGERD, 2016).

O sub atributo da consistência entre dispositivos se refere à necessidade de oferecer o máximo possível de padronização (similaridade) entre as interface do produto nos diferentes dispositivos (MAJRASHI; HAMILTON; L. UITDENBOGERD, 2016; MAJRASHI; HAMILTON; UITDENBOGERD, 2017; SEGERSTÅHL, 2008). Visa manter a coerência da experiência do usuário entre os dispositivos, facilitando operacionalidade, apreensibilidade e troca de dispositivos durante a tarefa (MAJRASHI; HAMILTON; UITDENBOGERD, 2018; WÄLJAS et al., 2010). Segundo Denis e Karsenty (2003), a consistência promove a continuidade de conhecimento e busca facilitar a recuperação, a reutilização e a adaptação do conhecimento construído pelo usuário a partir da interação com um ou mais dispositivos. Este é um requisito complexo que perpassa diversos aspectos da interface, incluindo (AABEL; ABEYWARNA, 2018; CELENTANO; DUBOIS, 2017; DEES, 2011; DENIS; KARSENTY, 2003; RINGBAUER, 2005; SEFFAH; FORBRIG; JAVAHERY, 2004; SEGERSTÅHL; OINAS-KUKKONEN, 2007; SKOV et al., 2015) : (a) consistência perceptiva, que visa a padronização visual e estrutural para facilitar o reconhecimento dos principais elementos da interface; (b) consistência léxica, focada nos termos e linguagem usados ao longo do sistema; (c) consistência sintática, que consiste em prover um passo a passo equivalente para completar a mesma tarefa em diferentes dispositivos; (d) consistência interativa, que aborda as modalidades interativas e a forma de manipular as interfaces (e) consistência semântica, que estabelece um eixo conceitual a partir do qual as interfaces dos diferentes dispositivos é implementada.

Por fim, a interoperabilidade entre dispositivos é um sub atributo essencial para produtos cross-device. Na norma ISO/IEC 25010 (ISO, 2011), a interoperabilidade está definida como um sub atributo para compatibilidade externa com outros produtos de software, que descreve o grau com o qual podem trocar informações e usar as informações que foram trocadas. Neste trabalho, este conceito foi trazido para o âmbito interno da usabilidade do conjunto. Assim, os dispositivos devem ser capazes de trocar informações entre si, permitindo que o usuário continue a tarefa de onde parou ao trocar de dispositivo (DENIS; KARSENTY, 2003). Segundo Tungare and Pérez-Quiñones (2009), o nível de suporte que o sistema oferece à continuidade afeta a carga mental percebida pelos usuários. Assim, o sistema deve permitir ao usuário retomar de modo fluído as tarefas interrompidas com a troca do dispositivo (AABEL; ABEYWARNA, 2018; MAJRASHI; HAMILTON; L. UITDENBOGERD, 2016; MAJRASHI; HAMILTON; UITDENBOGERD, 2017). A continuidade pode ser garantida por meio da sincronização automática de dados e conteúdos entre dispositivos (LUYTEN; CONINX, 2005; SEGERSTÅHL; OINAS-KUKKONEN, 2007; SKOV et al., 2015; WÄLJAS et al., 2010). Não deve ser exigido que os usuários lembrem de informações de um dispositivo para o outro (MAJRASHI; HAMILTON; L. UITDENBOGERD, 2016; MAJRASHI; HAMILTON; UITDENBOGERD, 2017). 


\subsubsection{Confiabilidade}

Diz respeito à capacidade do produto executar as funções especificadas sob condições especificadas por um período de tempo especificado (ISO, 2011). No multidevice, o sistema deve ser capaz de oferecer suporte confiável a uma estratégia envolvendo vários dispositivos (SHIN; BIOCCA, 2017). Como sub atributo, tem-se a disponibilidade, que descreve a capacidade do sistema de estar operacional e acessível quando seu uso for requisitado. No cross-device, depende da interconectividade entre os dispositivos, pois a comunicação entre eles é normalmente feita sem fio por meio da rede de internet. De acordo com Shin e Biocca (2017), os usuários esperam que as redes possam oferecer conectividade confiável e que o sistema esteja disponível em qualquer lugar e a qualquer momento, garantindo a usabilidade horizontal (MAJRASHI; HAMILTON; UITDENBOGERD, 2018).

\subsubsection{Segurança}

Este atributo descreve a capacidade de um produto de proteger as informações e os dados para que as pessoas ou outros sistemas tenham o grau de acesso compatível com seus níveis de autorização (ISO, 2011). No multi-device, a distribuição de tarefas e informações entre dispositivos leva a considerações sobre segurança que variam de acordo com o nível de privacidade do ambiente (HUTCHINGS; PIERCE, 2006). Segundo os autores, em ambientes particulares, os usuários podem distribuir suas tarefas e informações entre os diferentes dispositivos sem maiores preocupações. Em ambientes semi-privados e públicos, a forma de distribuição das tarefas e das informações entre os dispositivos deve permitir, de acordo com a situação, facilitar ou dificultar a sua visualização pelos espectadores, mantendo privado aquilo que é confidencial. A definição sobre aquilo que deve ser visto de forma compartilhada e aquilo que deve ser escondido dos espectadores é um fator que interfere na qualidade do uso em sistemas com múltiplos dispositivos (ALSOS; SVAN/ES, 2006; SKOV et al., 2015). Como consequência, no âmbito da segurança, deve ser permitido ao usuário obscurecer a apresentação de certos conteúdos em certos dispositivos, de acordo com a situação de uso.

\subsection{Modelo descritivo para qualidade cross-device}

Esta seção apresenta na tabela 4 uma síntese dos atributos discutidos anteriormente. Para cada um deles é proposto um conceito que descreve o seu significado no contexto multi-device. O propósito não é substituir as descrições providas pela norma ISO/IEC 25010 (ISO, 2011), mas complementá-las considerando as contribuições dos autores para abordagem da qualidade multi-device.

Tabela 4. Atributos de qualidade multi-device.

\begin{tabular}{|l|l|}
\hline ATRIBUTO & CONCEITO PARA O MULTI-DEVICE \\
\hline ADEQUAÇÃO FUNCIONAL & $\begin{array}{l}\text { Capacidade do produto prover uma distribuição adequada de funções e de } \\
\text { conteúdos entre os dispositivos. }\end{array}$ \\
\hline Adequação aos dispositivos & $\begin{array}{l}\text { Grau com que o produto utiliza dispositivos que adicionam valor à execução de } \\
\text { cada tarefa, facilitando sua realização. }\end{array}$ \\
\hline
\end{tabular}




\begin{tabular}{|c|c|}
\hline Adequação aos usuários & $\begin{array}{l}\text { Grau com que o produto adota dispositivos adequados aos usuários, considerando } \\
\text { familiaridade, contexto de uso, conhecimento técnico, habilidades, limitações, } \\
\text { preferências e possibilidades de personalização. }\end{array}$ \\
\hline Completude funcional & $\begin{array}{l}\text { Grau com que o produto oferece ao usuário pelo menos um dispositivo compatível } \\
\text { com as funções necessárias para cada tarefa no momento de sua execução. }\end{array}$ \\
\hline PORTABILIDADE & $\begin{array}{l}\text { Capacidade do produto ser usado na maior quantidade possível de plataformas, } \\
\text { ampliando seus canais de oferta. }\end{array}$ \\
\hline Adaptabilidade & $\begin{array}{l}\text { Capacidade da interface de estruturar, apresentar e executar tarefas de forma } \\
\text { compatível com os recursos de cada plataforma. }\end{array}$ \\
\hline USABILIDADE & $\begin{array}{l}\text { Capacidade do produto permitir que as metas sejam atingidas com eficácia, } \\
\text { eficiência e satisfação tanto quando ocorre o uso isolado de um dispositivo } \\
\text { (usabilidade vertical), quanto em casos de uso combinado de dispositivos } \\
\text { (usabilidade horizontal). }\end{array}$ \\
\hline Operacionalidade & $\begin{array}{l}\text { Capacidade do produto incorporar as modalidades interativas adequadas para cada } \\
\text { tarefa, considerando os níveis de atenção, carga cognitiva e carga física que podem } \\
\text { ser despendidos pelos os usuários de acordo com a situação de uso. }\end{array}$ \\
\hline Apreensibilidade & $\begin{array}{l}\text { Grau com que o produto oferece interfaces claras o suficiente para que os usuários } \\
\text { entendam como dados e funções estão distribuídos entre os dispositivos. }\end{array}$ \\
\hline $\begin{array}{l}\text { Consistência entre } \\
\text { dispositivos }\end{array}$ & $\begin{array}{l}\text { Capacidade do produto oferecer o máximo possível de padronização entre as } \\
\text { interfaces dos dispositivos, envolvendo aspectos perceptuais, léxicos, interativos e } \\
\text { semânticos. }\end{array}$ \\
\hline $\begin{array}{l}\text { Interoperabilidade entre } \\
\text { dispositivos }\end{array}$ & $\begin{array}{l}\text { Capacidade do produto promover sincronização de dados e conteúdos entre } \\
\text { dispositivos, apresentando as informações necessárias para os usuários } \\
\text { continuarem tarefas após a troca de dispositivo. }\end{array}$ \\
\hline CONFIABILIDADE & $\begin{array}{l}\text { Capacidade do produto oferecer suporte confiável à execução de uma tarefa com } \\
\text { múltiplos dispositivos. }\end{array}$ \\
\hline Disponibilidade & $\begin{array}{l}\text { Capacidade do produto permitir, quando requisitado, o uso individual ou conjunto } \\
\text { dos seus dispositivos. }\end{array}$ \\
\hline SEGURANÇA & $\begin{array}{l}\text { Capacidade do produto permitir, por questões de privacidade, que os usuários } \\
\text { obscureçam a apresentação de certos conteúdos em certos dispositivos de acordo } \\
\text { com a situação de uso. }\end{array}$ \\
\hline
\end{tabular}

Além dos conceitos, este trabalho também propõe um modelo para a organização dos atributos em dois grupos, conforme mostrado na figura 6 . 0 primeiro grupo está ligado à qualidade da arquitetura funcional do produto, que estabelece como o produto dará suporte à atividade do usuário em diferentes dispositivos. Neste grupo, foi incluído o atributo da adequação funcional e todos os seus sub-atributos. Também foi incluído o atributo da confiabilidade e seu sub atributo da disponibilidade, que relacionam-se à requisitos técnicos da infraestrutura de conexão para viabilizar o modelo conceitual proposto.

O segundo grupo aborda a qualidade de interações e interfaces, que representa a forma escolhida para dar suporte às funções identificadas na arquitetura funcional. Neste grupo, foi incluído o atributo da portabilidade e seu sub atributo da adaptabilidade, focados na qualidade do ajuste das interfaces para cada dispositivo. Também foi incluído o atributo da usabilidade e seus sub-atributos, que abordam o processo de uso e contrabalanceiam uma possível visão técnica focada em dispositivos. Por fim, foi 
incluído o atributo da segurança, cada vez mais importante à medida que os sistemas computacionais detêm um volume de informações crescente sobre os usuários.

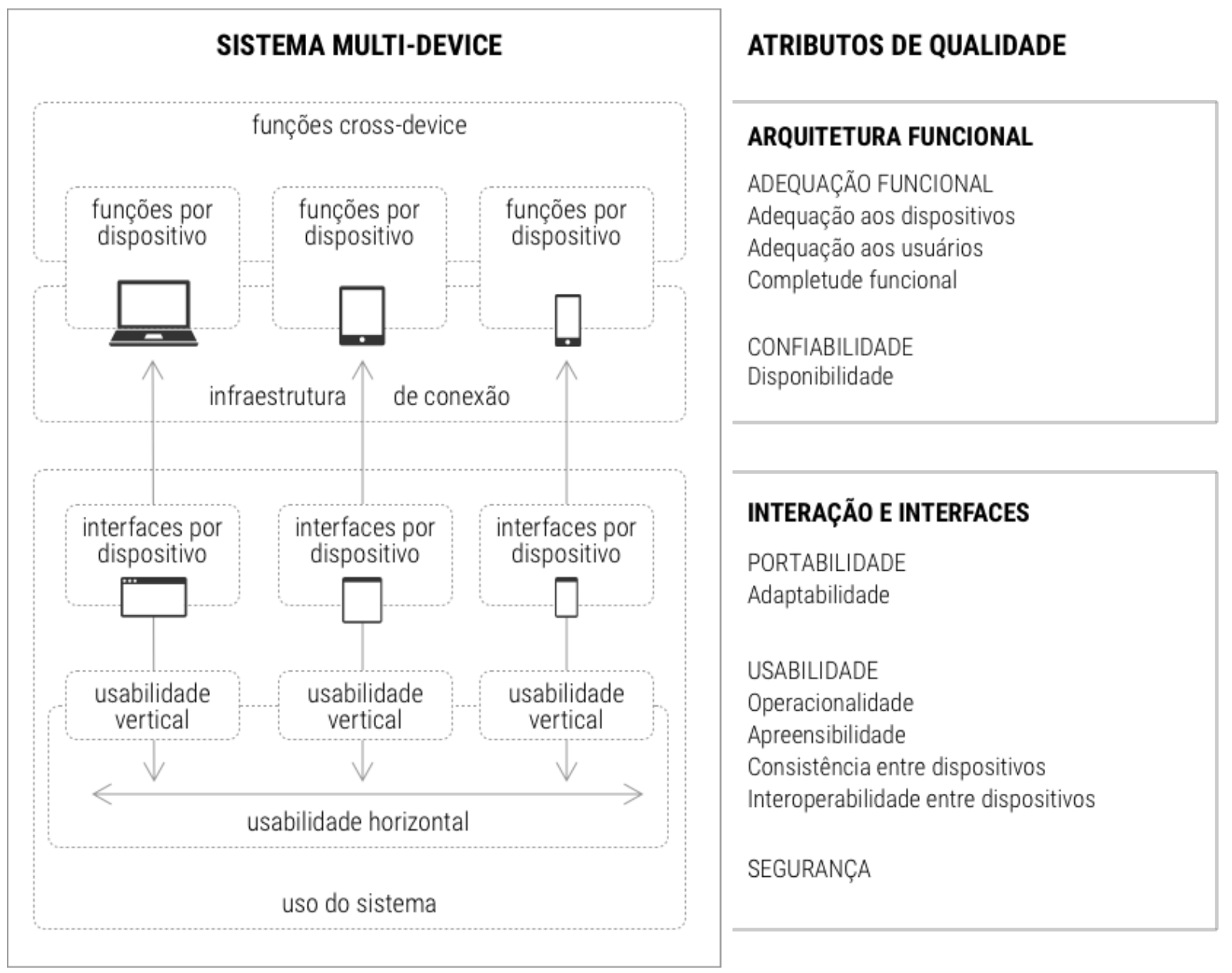

Figura 6 - Modelo de qualidade multi-device (fonte: autores).

\section{Discussão}

Aqualidade em sistemas multi-device torna-se relevante à medida que se ampliam o número de dispositivos disponíveis. Embora o uso combinado de dispositivos esteja se popularizando apenas agora, reflete a forma como as pessoas agem com os diversos objetos do dia a dia. Segundo Bodker e Andersen (2005), a mediação das atividades humanas é um processo complexo por natureza, baseado em uma teia de mediadores que podem ser ligados em cadeia, organizados em níveis ou usados ao mesmo tempo de acordo com a finalidade. Desta forma, abordar a qualidade multi-device consiste em criar condições para desenvolver a mediação múltipla no âmbito computacional. Esse é um tópico importante para o design e para a ergonomia considerando a perspectiva da computação como um componente ubíquo (WEISER, 1999), que pode ser integrado a qualquer objeto e ao próprio ambiente arquitetônico.

Considerando estas questões, o foco nos atributos de qualidade visa estabelecer parâmetros que dêem suporte ao processo de projeto multi-device. Estes atributos podem ser usados como heurísticas que visam estimular o pensamento criativo durante a concepção do produto ou a avaliação do resultado obtido. Uma heurística pode ser 
entendida como uma estratégia cognitiva que busca diminuir a complexidade de um processo de tomada de decisão, apoiando a solução de problemas do seu estado inicial ao final (ROMANYCIA; PELLETIER, 1985). Assim, ao oferecer parâmetros sobre o que deve ser contemplado pela arquitetura funcional, pelas interações e pelas interfaces, os atributos direcionam o processo de tomada de decisões durante o projeto.

Para clarificar a relação entre os atributos propostos e o processo de projeto, podese tomar como base o trabalho de Garrett (2011), focado na abordagem da experiência do usuário em sistemas digitais. Segundo o autor, há cinco fases de projeto, que vão da concepção abstrata à maturidade e materialização: (a) identificação das necessidades do usuário e objetivos do projeto; (b) especificação das funcionalidades que o produto deve conter para contemplar as necessidades e objetivos; (c) design de interação para definição dos fluxos de aplicação capazes de facilitar as tarefas do usuário e sua interação com as funcionalidades; (d) design da interface e da informação para facilitar a interação e a compreensão do processo; (e) design visual para tratamento gráfico dos elementos da interface.

Considerando estas fases, é possível usar os atributos de adequação funcional e confiabilidade como heurísticas para a fase de especificações funcionais. Por outro lado, os atributos de portabilidade, usabilidade e segurança impactam no processo de concretização das especificações funcionais, podendo atuar como heurísticas para os processos de design de interação, de interface, de informação e visual. Esta relação é demonstrada na figura 7.

ELEMENTOS DA EXPERIÊNCIA DO USUÁRIO

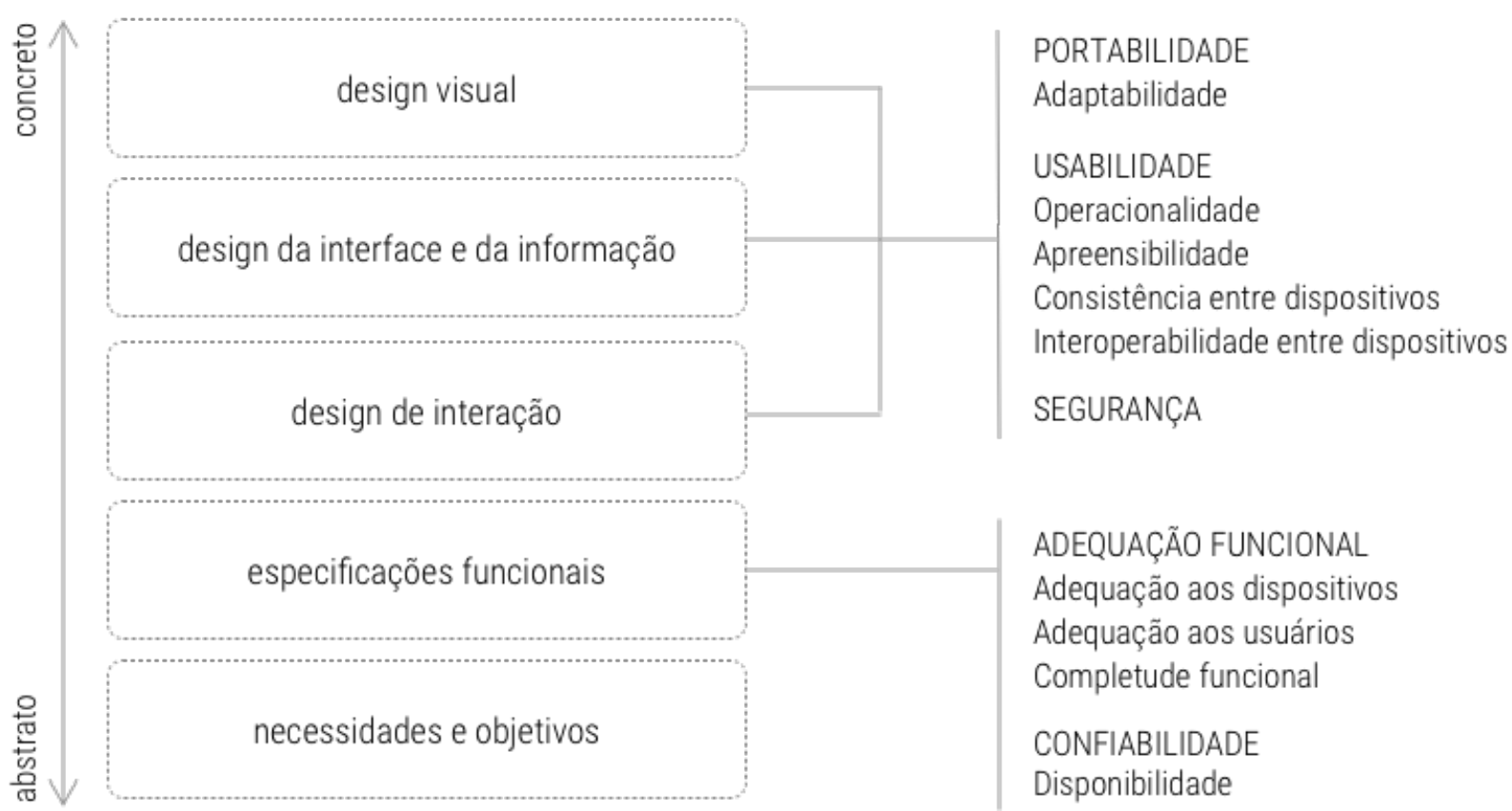

Figura 7 - Elementos da experiência do usuário e atributos de qualidade multi-device (fonte: autores).

\section{Conclusão}

Este trabalho apresentou um modelo de atributos de qualidade para produtos de software multi-device. Sua construção foi baseada em uma revisão sistemática 
focada na usabilidade, ergonomia e experiência do usuário em sistemas multi-device. Foram identificados 21 trabalhos que abordam aspectos que influenciam na qualidade multi-device. Estes aspectos foram consolidados para se obter uma padronização de nomenclatura. Além disso, foram organizados em um modelo que estabelece parâmetros para processo de projeto e avaliação.

Considerando o fato da interação multi-device ser recente, a principal contribuição deste trabalho é consolidar em uma estrutura didaticamente organizada os conceitos relacionados ao multi-device, bem como seus parâmetros de qualidade. Cabe ao projetista se apropriar das tecnologias disponíveis e aplicá-las de modo adequado na concepção de sistemas ergonômicos que atendam às necessidades dos usuários. Tal tarefa, no entanto, não é simples. A pesquisa feita por Dong et al. (2016) com profissionais de projeto identificou que muitas vezes é difícil para estes profissionais entenderem e comunicarem como vários dispositivos funcionarão juntos sob diferentes circunstâncias. Em especial, profissionais com um perfil menos tecnológico se vêem diante do desafio de projetar ou de avaliar sistemas com múltiplos dispositivos sem conhecer os atributos de qualidade que precisam ser observados.

Por fim, como trabalho futuro, sugere-se a criação de métricas para cada um dos atributos e dos sub-atributos propostos. Com base nestas métricas, o modelo poderá ser ampliado para permitir a mensuração da qualidade. 


\section{REFERÊNCIAS}

AABEL, B.; ABEYWARNA, D. Digital Cross-Channel Usability Heuristics: Improving the Digital Health Experience. Journal of Usability Studies, v. 13, n. 2, 2018.

ALSOS, O. A.; SVANAES, D. Interaction Techniques for Using Handhelds and PCs Together in a Clinical Setting. Proceedings of the 4th Nordic Conference on Humancomputer Interaction: Changing Roles. Anais...: NordiCHI '06.New York, NY, USA: ACM, 2006.

BØDKER, S.; ANDERSEN, P. B. Complex Mediation. Hum. Comput. Interact., v. 20, n. 4, p. 353-402, 2005.

CALVARY, G. et al. A Unifying Reference Framework for multi-target user interfaces. Interacting with computers, v. 15, n. 3, p. 289-308, 2003.

CELENTANO, A.; DUBOIS, E. Interaction-in-the-large vs interaction-in-the-small in multi-device systems. Proceedings of the 12th Biannual Conference on Italian SIGCHI Chapter. Anais...ACM, 18 set. 2017.

DEES, W. Usability of Nomadic User Interfaces. (J. A. Jacko, Ed.)Human-Computer Interaction. Towards Mobile and Intelligent Interaction Environments. Anais...: Lecture Notes in Computer Science. In: INTERNATIONAL CONFERENCE ON HUMAN-COMPUTER INTERACTION. Springer Berlin Heidelberg, 2011.

DENIS, C.; KARSENTY, L. Inter-Usability of Multi-Device Systems - A Conceptual Framework. In: Multiple User Interfaces. [s.I.] John Wiley \& Sons, Ltd, 2003. p. 373-385.

DONG, T.; CHURCHILL, E. F.; NICHOLS, J. Understanding the Challenges of Designing and Developing Multi-Device Experiences. Proceedings of the 2016 ACM Conference on Designing Interactive Systems. Anais...ACM, 2016.

FLORINS, M.; VANDERDONCKT, J. Graceful degradation of user interfaces as a design method for multiplatform systems. Proceedings of the 9th international conference on Intelligent user interfaces. Anais...ACM, 2004.

GARRETT, J. J. The Elements of User Experience: User-centered Design for the Web and Beyond. [s.l.] New Riders, 2011.

HARPER, R. Being Human: Human-computer Interaction in the Year 2020. [s.l.] Microsoft Research, 2008.

HUTCHINGS, H. M.; PIERCE, J. S. Understanding the whethers, hows, and whys of divisible interfaces. Traducao Dipartimento di Informatica; Universita di Milano Universita Ca' Foscari Venezia Dipartimento di Info. e Comunicazione; Accademia di Belle Arti di Venezia; DELOS. [s.l: s.n.]. v. 2006

ISO. ISO/IEC 25010. Systems and Software Engineering-Systems and Software Quality Requirements and Evaluation (SQuaRE)_System and Software Quality Models, 2011. 
JOKELA, T.; OJALA, J.; OLSSON, T. A Diary Study on Combining Multiple Information Devices in Everyday Activities and Tasks. Proceedings of the 33rd Annual ACM Conference on Human Factors in Computing Systems. Anais...: CHI '15.New York, NY, USA: ACM, 2015

KITCHENHAM, B.; CHARTERS, S. Guidelines for performing Syst Software Engineeri. [s.I.] Keele University and Durham University Joint Report, 2007.

LEVIN, M. Designing Multi-Device Experiences: An Ecosystem Approach to User Experiences across Devices. [s.I.] "O'Reilly Media, Inc.", 2014.

LUYTEN, K.; CONINX, K. Distributed user interface elements to support smart interaction spaces. Seventh IEEE International Symposium on Multimedia (ISM'05). Anais...dez. 2005.

MAJRASHI, K.; HAMILTON, M.; L. UITDENBOGERD, A. Cross-Platform Cross-Cultural User Experience. : Electronic Workshops in Computing. In: BCS HUMAN COMPUTER INTERACTION CONFERENCE 2016. BCS Learning \& Development, 2016.

MAJRASHI, K.; HAMILTON, M.; UITDENBOGERD, A. L. The Ubiquitous Device Transition Experience of the Mobile User. Proceedings of the 14th EAl International Conference on Mobile and Ubiquitous Systems: Computing, Networking and Services. Anais...ACM, 7 nov. 2017.

MAJRASHI, K.; HAMILTON, M.; UITDENBOGERD, A. L. Task Continuity and Mobile User Interfaces. Proceedings of the 17th International Conference on Mobile and Ubiquitous Multimedia. Anais...ACM, 25 nov. 2018.

NEATE, T.; EVANS, M.; JONES, M. Enhancing interaction with dual-screen television through display commonalities. Proceedings of the 2017 ACM International, 2017.

NGUYEN, T.-D.; VANDERDONCKT, J.; SEFFAH, A. Generative patterns for designing multiple user interfaces. Proceedings of the International Conference on Mobile Software Engineering and Systems. Anais...ACM, 14 maio 2016.

NIGAY, L.; COUTAZ, J. A Design Space for Multimodal Systems: Concurrent Processing and Data Fusion. Proceedings of the INTERACT' 93 and CHI '93 Conference on Human Factors in Computing Systems. Anais...: CHI '93.New York, NY, USA: ACM, 1993.

ÖQUIST, G.; GOLDSTEIN, M.; CHINCHOLLE, D. Assessing Usability across Multiple User Interfaces. In: SEFFAH, A.; JAVAHERY, H. (Eds.). . Multiple User Interfaces. Chichester, UK: John Wiley \& Sons, Ltd, 2005. p. 325-349.

PATERNÒ, F. Technological platforms, convergence and adaptation of interactive contents. In: PAZOS-ARIAS, J. J.; KLOOS, C. D.; NORES, M. L. (Eds.). . Personalization of Interactive Multimedia Services: A Research and Development Perspective. Hauppauge: Nova Science Publishers, 2009. p. 25-48.

PATERNÒ, F.; SANTORO, C. A Logical Framework for Multi-device User Interfaces. Proceedings of the 4th ACM SIGCHI Symposium on Engineering Interactive Computing 
Systems. Anais...: EICS '12.New York, NY, USA: ACM, 2012.

RINGBAUER, B. SMART HOME CONTROL VIA PDA. In: SLOANE, A. (Ed.). . Home-Oriented Informatics and Telematics. IFIP International Federation for Information Processing. Boston: Kluwer Academic Publishers, 2005. v. 178p. 101-119.

ROMANYCIA, M. H. J.; PELLETIER, F. J. What is a heuristic? Computational Intelligence. An International Journal, v. 1, n. 1, p. 47-58, 1985.

SEFFAH, A.; FORBRIG, P.; JAVAHERY, H. Multi-devices "Multiple" user interfaces: development models and research opportunities. The Journal of systems and software, v. 73, n. 2, p. 287-300, 2004.

SEGERSTÅHL, K. Utilization of Pervasive IT Compromised?: Understanding the Adoption and Use of a Cross Media System. Proceedings of the 7th International Conference on Mobile and Ubiquitous Multimedia. Anais...: MUM '08.New York, NY, USA: ACM, 2008.

SEGERSTÅHL, K.; OINAS-KUKKONEN, H. Distributed User Experience in Persuasive Technology Environments. (Y. de Kort et al., Eds.)Persuasive Technology. Anais...: Lecture Notes in Computer Science. In: INTERNATIONAL CONFERENCE ON PERSUASIVE TECHNOLOGY. Springer Berlin Heidelberg, 2007.

SHIN, D.-H.; BIOCCA, F. Explicating user behavior toward multi-screen adoption and diffusion: User experience in the multi-screen media ecology. Internet Research, v. 27, n. 2, p. 338-361, 2017.

SKOV, M. B. et al. Investigating Cross-Device Interaction Techniques: A Case of Card Playing on Handhelds and Tablets. Proceedings of the Annual Meeting of the Australian Special Interest Group for Computer Human Interaction. Anais...: OzCHI '15. New York, NY, USA: ACM, 2015.

TUNGARE, M.; PÉREZ-QUINONES, M. A. Mental Workload at Transitions between Multiple Devices in Personal Information Management. Personal Information Management Workshop, ASIST Vancouver. Anais...pimworkshop.org, 2009.

VAN DAM, A. Beyond WIMP. IEEE computer graphics and applications, v. 20, n. 1, p. 50-51, jan. 2000.

WÄLJAS, M. et al. Cross-platform Service User Experience: A Field Study and an Initial Framework. Proceedings of the 12th International Conference on Human Computer Interaction with Mobile Devices and Services. Anais...: MobileHCl '10.New York, NY, USA: ACM, 2010.

WEISER, M. The computer for the 21 st century. ACM SIGMOBILE Mobile Computing and Communications Review, v. 3, n. 3, p. 3-11, 1999.

ZAGERMANN, J. et al. Studying the Benefits and Challenges of Spatial Distribution and Physical Affordances in a Multi-device Workspace. Proceedings of the 16th International Conference on Mobile and Ubiquitous Multimedia. Anais...: MUM '17.New York, NY, USA: ACM, 2017. 